\title{
Research on the Coordinated Control of Photovoltaic Microgrid Based on Micro Gas Turbine
}

\author{
G B Zeng \\ Department of Power Engineering, Anhui Electrical \\ Engineering Professional Technique College, Hefei, Anhui \\ 230051, China
}

\begin{abstract}
The hybrid microgrid can make full use of the distributed generation to supplement the large power grid, but different power sources need to be coordinated before supplying more stable power. This study proposed an improved coordinated control strategy for the heterogeneous power in the hybrid microgrid. In this strategy, the reference power of the gas turbine was calculated by the predictive algorithm instead of the traditional reference power calculation method. Then, the simulation experiment was carried out. The results showed that the micro gas turbine provided the power when the photovoltaic output power was insufficient to bear the local load due to the decrease of light intensity, and the gas turbine under the improved control strategy provided the power faster; the voltage fluctuation of the distribution network bus caused by the sudden change of illumination was smaller and lasted for a shorter time under the improved coordinated control strategy; the fifth and seventh harmonics generated by the voltage fluctuation were smaller under the improved strategy.
\end{abstract}

\section{INTRODUCTION}

The power supply of the traditional power grid system is in the form of single centralization; thus, once there is a problem in some areas, it will affect the stability of the whole power grid. Moreover, the scale of the power grid increases with the increase of the power generation scale; as a result, the edge of the power grid is far away from the power generation center, and the current feeder loss is serious [1]. Compared with the traditional power grid, the distributed power grid [16] is more flexible in the power supply. Even if there is a problem in the distributed power grid, it can be disconnected in time to ensure the stability of the main grid. Moreover, the distributed grid can flexibly add microgrid at the edge to reduce the feeder loss at the edge of the grid, and this kind of power supply form is quite suitable for the existing renewable energy, such as photovoltaic power generation, wind power generation, etc. [2]. Although the power provided by the renewable energy in a microgrid is weaker than that in the main grid, it can play a complementary role. As a part of the distributed power grid, microgrid also has some characteristics of the distributed grid: the power generation in the grid is distributed, and the grid with different types of power can form a larger grid [17].

${ }^{*}$ Corresponding Author: z_guob@yeah.net 
The power grid composed of different types of power can make up for the defects of their respective power. However, due to different types, the power supply between them will not match. Therefore, it is necessary to have appropriate coordinated control strategies [3] to bridge them and reduce the instability of microgrid. Xia et al. [4] proposed a control method of the parallel bidirectional power converter based on decentralized control to provide good voltage support for direct current microgrid and verified the reliability of the method by simulation and actual hardware experiments. Zhou et al. [5] proposed a microgrid cluster structure and its autonomous coordinated control strategy and verified through the simulation and actual hardware experiment that the control strategy could effectively stabilize the microgrid. This paper proposed an improved coordinated control strategy for the heterogeneous power in the hybrid microgrid. In this strategy, the reference power of the gas turbine was calculated by the predictive algorithm instead of the traditional reference power calculation method. Then, the simulation experiment was carried out.

\section{MICRO GAS TURBINE - PHOTOVOLTAIC MICROGRID}

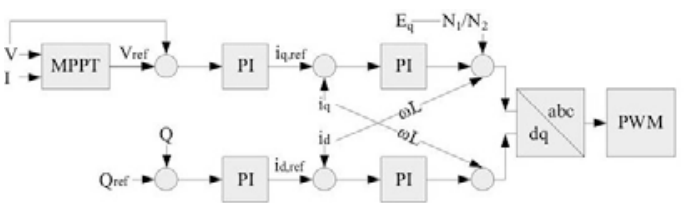

Control framework of photovoltaic inverter

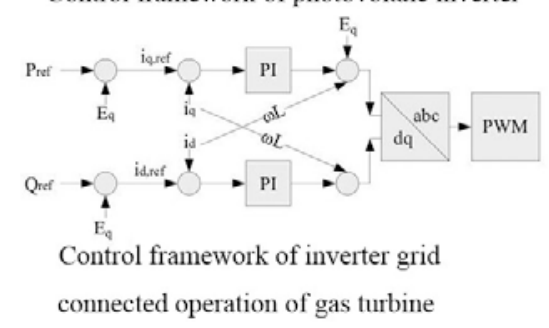

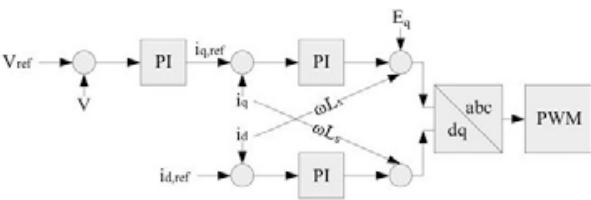

Control framework of gas turbine rectifier

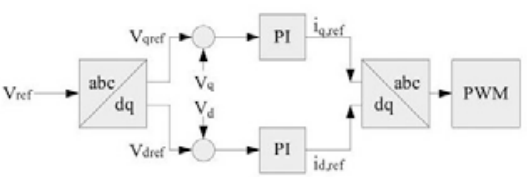

Control framework of inverter isolated

island operation of gas turbine

Figure 1 The power control framework of different power in the hybrid microgrid

In the process of using different types of power in the microgrid, whether it is in grid operation or island operation [7], the power in the grid will fluctuate when the operation state changes. If not controlled, the fluctuation will spread to the whole grid, reducing the stability of the grid.

As shown in Figure 1, the photovoltaic inverter obtains the reference value of the direct current voltage $\left(V_{r e f}\right)$ by Maximum Power Point Tracking (MPPT) algorithm [8]. $\left(i_{q, r e f}\right)$, the current reference value of the q axis is obtained by processing the difference between the actual direct current voltage $\mathrm{V}$ and $V_{\text {ref }}$ by PI. $i_{d, r e f}$, the current reference value of the $\mathrm{d}$ axis, is obtained by processing the difference between the reactive power reference value $Q_{r e f}$ and the actual reactive power $\mathrm{Q}$. The difference between the reference current and actual current of $\mathrm{q}$ and $\mathrm{d}$ axes is processed by PI, and then the voltage difference of the $\mathrm{q}$ axis is combined with the $q$ axis potential and $d$ axis current that have been processed to obtain the q axis voltage reference value needed by the inverter. 
The voltage reference values of the $\mathrm{q}$ and $\mathrm{d}$ axes are transformed into three-phase voltage reference values to trigger Pulse-Width Modulation (PWM) signal [9] to regulate the inverter.

The process of the micro gas turbine is similar to the previous text. Firstly, the $q$ axis reference current is obtained by processing the difference between the actual voltage and the reference voltage of the rectifier with PI. Then the reference current of the $q$ and $d$ axes is compared with the actual current, respectively. After the difference is processed by PI, the voltage difference of the $\mathrm{q}$ axis is combined with the $\mathrm{q}$ axis potential and the processed $\mathrm{d}$ axis current. The voltage difference of the $d$ axis is combined with the processed $q$ axis current, and finally, the voltage reference values of the $q$ and $d$ axes are obtained.

\section{COORDINATED CONTROL STRATEGY}

\subsection{Traditional Coordinated Control Strategy}

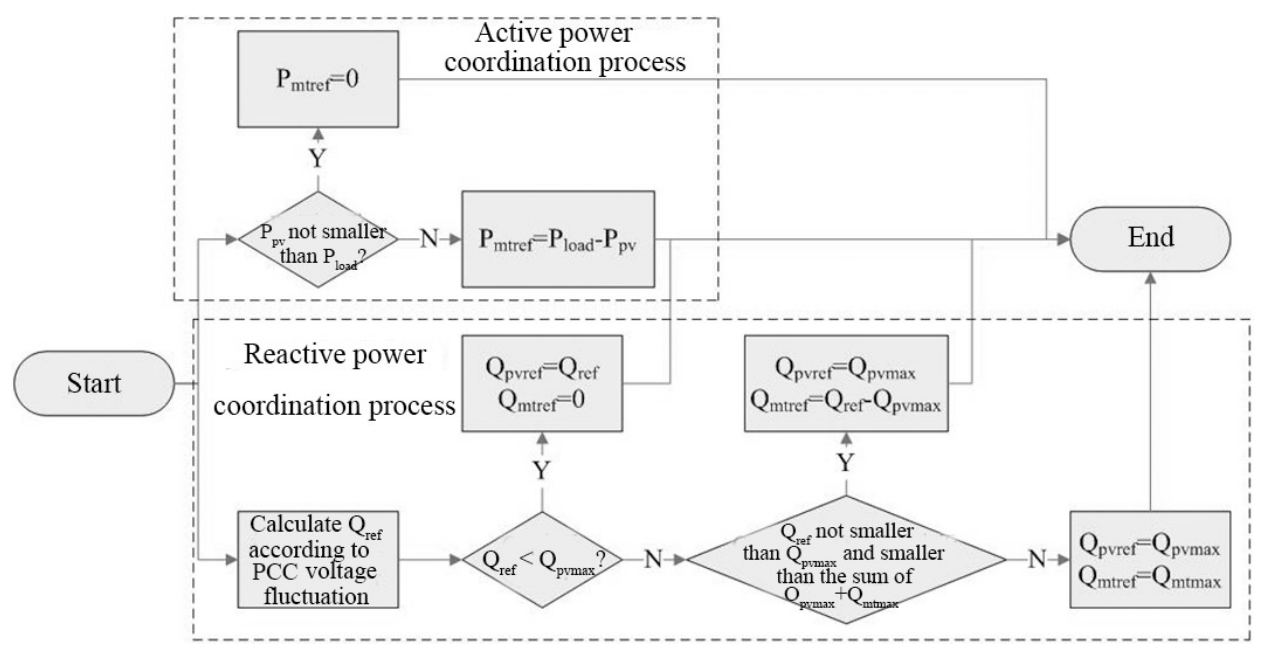

Figure 2 The traditional coordinated control flow of the hybrid microgrid

In the hybrid microgrid, the photovoltaic array is the main power output, and the gas turbine is the auxiliary. Once the output of the photovoltaic array is unstable due to environmental change, the gas turbine is started to supplement it. In this process, they need to coordinate to reduce grid instability caused by power load change and reduce the waste of the micro grid power [6]. As shown in Figure 2, the traditional coordinated control strategy starts from active and reactive power, and the control of photovoltaic power generation is realized through the photovoltaic inverter control framework described above; the gas turbine regulates $P_{\text {mtref }}$ according to the photovoltaic output power.

In the process of the active power coordination, the photovoltaic power $P_{p v}$ obtained by sensors in the grid is compared with the load demand power $P_{\text {load }}$ in the grid. 
If $P_{p v}$ is not smaller than $P_{l o a d}$, i.e., the photovoltaic power generation can satisfy the load demand of the grid, then the assistance of gas turbine is not needed, i.e., the active reference power $P_{\text {mtref }}$ of the gas turbine is 0 ; if $P_{p v}$ is smaller than $P_{\text {load }}$, then the assistance of gas turbine is needed, and $P_{m t r e f}$ is the difference of $P_{p v}$ and $P_{\text {load }}$. After obtaining $P_{m t r e f}$, it is used for the control of the gas turbine.

The coordinated control of reactive power is to reduce the voltage fluctuation at the grid connection point and improve the stability. The process is as follows. The voltage fluctuation $\Delta U_{p c c}$ at the point of common coupling (PCC) at the grid connection [10] is collected by using sensors. Then $Q_{r e f}$, the reference value of reactive power that is used for stabilizing PCC voltage, is calculated, and the calculation formula is given in Eq. 1:

$$
\left\{\begin{array}{c}
Q_{\text {ref }}=\frac{\Delta V\left|\Delta U_{p c c}\right|}{S_{\text {nom }}} \\
\Delta V=\frac{V_{\text {max }}-V_{\text {min }}-Z}{2}
\end{array}\right.
$$

where $S$ is the rated capacity of the inverter [11], $V_{\max }$ and $V_{\min }$ are the maximum and minimum values of voltage fluctuation within the rated capacity range of the inverter, and $Z$ is the "dead zone" within the voltage fluctuation range [19], $Q_{r e f}=0$ when the voltage fluctuates in this range. Then whether $Q_{r e f}$ is smaller than $Q_{p v r e f}$ is determined. If it is, then $Q_{\text {pvref }}=Q_{\text {ref }}, Q_{\text {mtref }}=0$; if not, then whether $Q_{\text {ref }}$ is within $\left[Q_{\text {pvmax }}, Q_{\text {pvmax }}+Q_{\text {mtmax }}\right)$ is determined. If it is, then $Q_{\text {pvref }}=Q_{\text {pvmax }}, Q_{\text {mtref }}=Q_{\text {ref }}-Q_{\text {pvmax }}$; if not, then $Q_{\text {pvref }}=$ $Q_{\text {pvmax }}, Q_{\text {mtref }}=Q_{\text {mtmax }}$. After obtaining $Q_{\text {pvref }}$ and $Q_{m \text { tref }}$, they are used for controlling photovoltaic and gas turbine. The above process can be referred to Eq. 2:

$$
\left\{\begin{array}{c}
Q_{p v \max }=\sqrt{S_{p v}^{2}-P_{p v}^{2}} \\
Q_{\text {mtmax }}=\sqrt{S_{m t}^{2}-P_{m t r e f}^{2}}
\end{array}\right.
$$

where $Q_{\text {pvmax }}$ and $Q_{\text {mtmax }}$ are the maximum reactive power of photovoltaic array and gas turbine respectively and $S_{p v}$ and $S_{m t}$ are the rated capacity of inverter for photovoltaic array and gas turbine respectively [18].

\subsection{Improved Coordinated Control Strategy}

The traditional strategy can effectively stabilize the microgrid. However, in practical applications, the output of the photovoltaic array is very sensitive to environmental changes and changes rapidly. When gas turbine generates power, it relies on not only rectifiers and inverters but also the complex turbine control adjustment. In the face of rapid changes in the output of the photovoltaic array, the response of the gas turbine is slightly slow [12]. 
In the period of gas turbine delay, the photovoltaic array cannot be fully assisted, which leads to a decrease of grid stability. In this study, the power of the gas turbine was predicted to deal with the problem of the slow dynamic response of the gas turbine. In short, in the traditional coordinated control process, $P_{\text {mtref }}$ of gas turbine was predicted by the prediction algorithm [13], and the prediction formula is given in Eq. 3:

$$
\left\{\begin{array}{c}
G(z)=\frac{1-z^{-L}}{L\left(1-z^{-L}\right)}+\frac{T_{p} M^{2}}{T_{S} N^{2}} \cdot \frac{\left(1-z^{-\frac{N}{M}}\right)^{2}}{1-z^{-1}} \sum_{i=1}^{M-1} \omega_{i} z^{-\frac{N(i-1)}{M}} \\
T_{p}=\frac{(L-1) T_{S}}{2}+\tau
\end{array}\right.
$$

where $\mathrm{z}$ represents the output sampling sequence of photovoltaic array collected by sensors, $G(z)$ represents the predicted value, $L$ is the predictive step length, $T_{s}$ is the sampling period, $T_{p}$ is the predictive lead, $\mathrm{N}$ is the number of sampling points, $M$ is the number of regions divided in the sampling sequence, $\omega_{i}$ is the the weight of the data difference corresponding to adjacent regions, and $\tau$ is the time delay of the gas turbine.

\section{SIMULATION EXPERIMENT}

\subsection{Experimental Environment}

In this study, the simulation experiment was carried out on the coordinated control strategy of the microgrid isolated island operation stability using MATLAB software [14]. The experiment was carried out on a laboratory server with configurations of Windows7 system, I7 processor, and $16 \mathrm{G}$ memory.

\subsection{Experimental Setup}

The structure of the simulation model is shown in Figure 3. The two power generation systems were connected with the distribution network bus, and the distribution network bus was connected with the local load and large power grid as PCC. The dotted arrow in Figure 3 indicates the microgrid power information collected by the sensors transmitted to the control center; the solid arrow represents the PWM control signal fed back by the control center after calculating the power information [20]. In the simulation experiment, the related parameters for reference are shown in Table 1. 


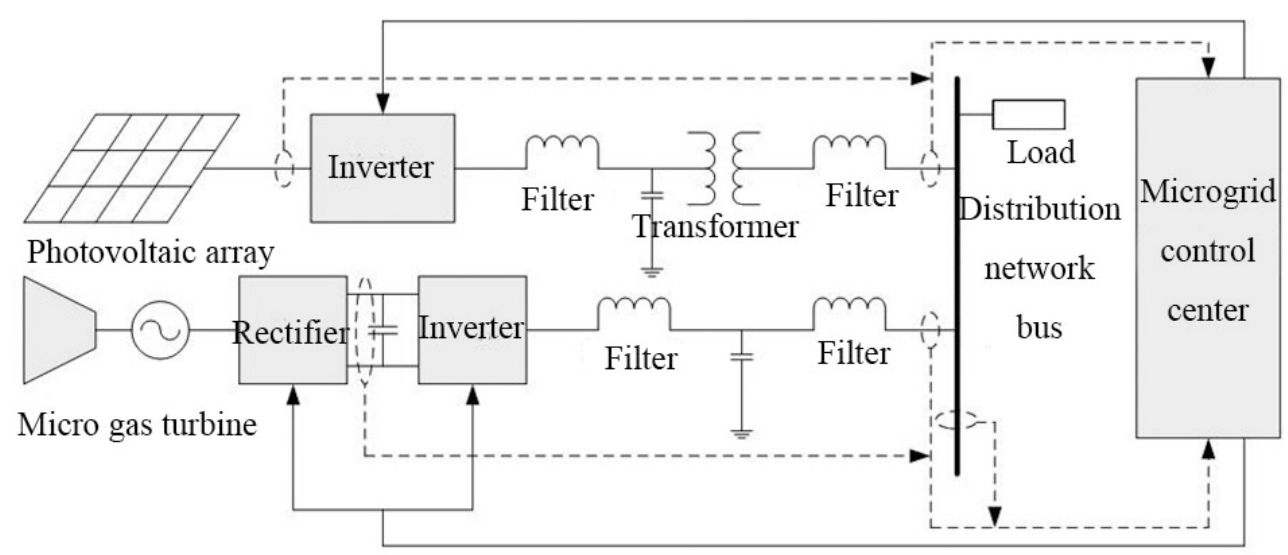

Figure 3 The basic structure of the hybrid microgrid coordinated control

Table 1 Parameters setting of the hybrid microgrid

\begin{tabular}{|c|c|c|c|}
\hline Parameter & Numerical value & Parameter & Numerical value \\
\hline $\begin{array}{l}\text { Rated power of } \\
\text { photovoltaic array }\end{array}$ & $20 \mathrm{~kW}$ & $\begin{array}{l}\text { Rated voltage of } \\
\text { photovoltaic array }\end{array}$ & $380 \mathrm{~V}$ \\
\hline $\begin{array}{l}\text { Rated power of gas } \\
\text { turbine }\end{array}$ & $30 \mathrm{~kW}$ & $\begin{array}{l}\text { Rated voltage of gas } \\
\text { turbine }\end{array}$ & $380 \mathrm{~V}$ \\
\hline Line impedance & $0.642 \Omega / \mathrm{km}$ & $\begin{array}{l}\text { Rated capacity of } \\
\text { rectifier }\end{array}$ & $50 \mathrm{~kW}$ \\
\hline $\begin{array}{l}\text { Sampling points of the } \\
\text { prediction algorithm }\end{array}$ & 800 & $\begin{array}{l}\text { Rated capacity of } \\
\text { inverter }\end{array}$ & $50 \mathrm{~kW}$ \\
\hline $\begin{array}{l}\text { Sampling period of the } \\
\text { prediction algorithm }\end{array}$ & $0.5 \mathrm{~s}$ & $\begin{array}{l}\text { Sampling region } \\
\text { number of the } \\
\text { prediction algorithm }\end{array}$ & 15 \\
\hline $\begin{array}{l}\text { Response delay of gas } \\
\text { turbine }\end{array}$ & $10 \mathrm{~s}$ & $\begin{array}{l}\text { Step length of the } \\
\text { prediction algorithm }\end{array}$ & 800 \\
\hline
\end{tabular}

\subsection{Experimental Methods}

Firstly, the model was built in MATLAB according to the hybrid microgrid structure shown above. The traditional coordinated control and improved coordinated control strategies were loaded into the microgrid control center module, and then the simulation experiment started. The process flow is as follows. Two power generation systems were turned on at the same time and connected to the distribution network bus [15], in which the power required by the local load of the microgrid was $18 \mathrm{kw}$. The variation curve of the illumination intensity of the photovoltaic power generation system is shown in Figure 4. The initial illumination intensity was $800 \mathrm{~W} / \mathrm{m} 2$. After $10 \mathrm{~s}$, the illumination intensity suddenly dropped to $400 \mathrm{~W} / \mathrm{m} 2$ and maintained until $20 \mathrm{~s}$. After the two power generation systems were connected to the distribution network, the moment when the photovoltaic array began to receive light was taken as the initial time. The light intensity changed according to the curve in Figure 4 in the next $20 \mathrm{~s}$, and the power changes in the grid under the two coordinated control strategies were recorded respectively. 


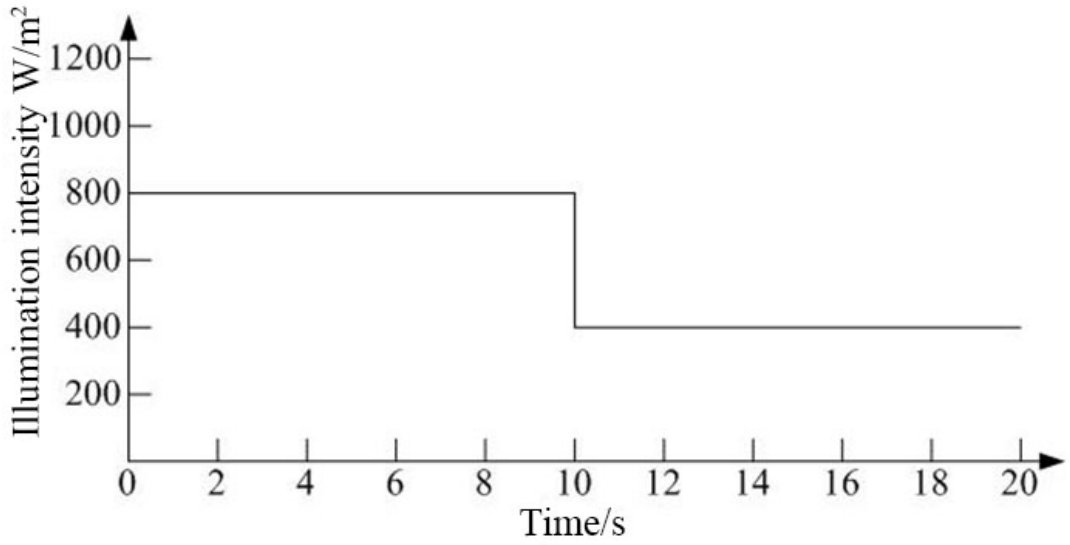

Figure 4 Variation curve of illumination intensity in the simulation experiment

\subsection{Experimental Results}

As shown in Figure 5, the output power suddenly reduced at $10 \mathrm{~s}$, then the micro gas turbine started to output electric energy to ensure the power required by the load, and the output power increased first and tended to be stable. Under the two coordinated control strategies, the variation trend of the output power of the photovoltaic and gas turbine was similar, especially the change of photovoltaic output power nearly coincided. However, it was seen from Figure 5 that the output power of the result was that the non-negligible dynamic response of the gas turbine made the improved control strategy based on the prediction algorithm start the gas turbine ahead of time to reduce the delay.

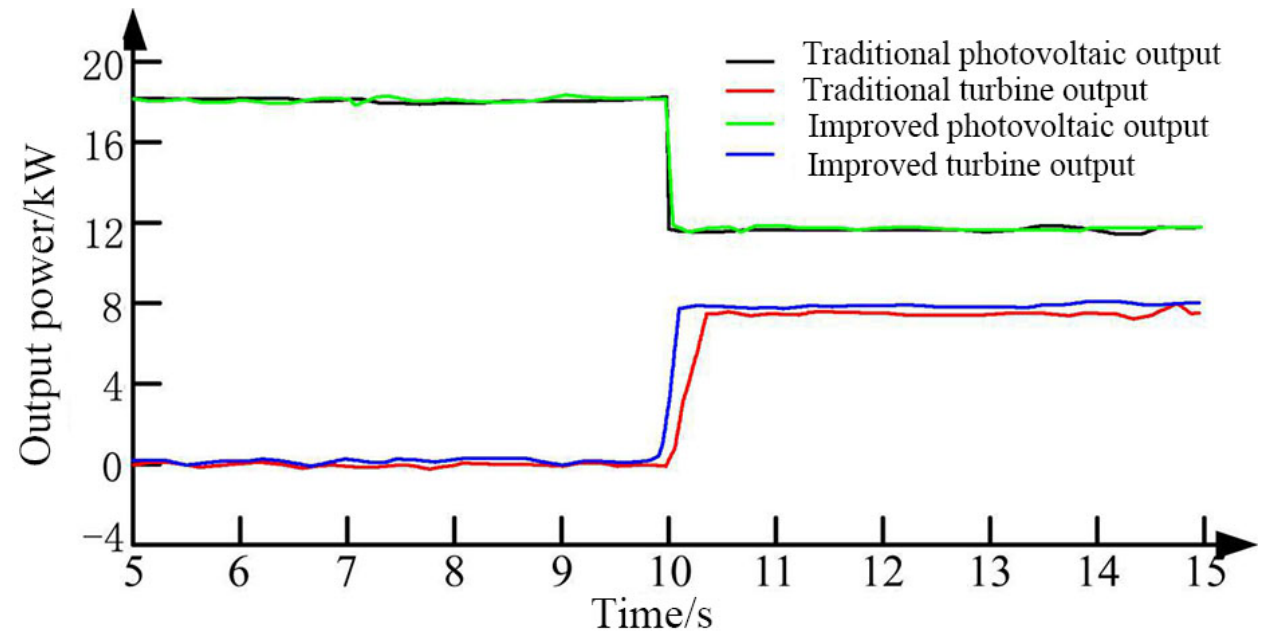

Figure 5 The output power variation of the hybrid microgrid under two coordinated control 


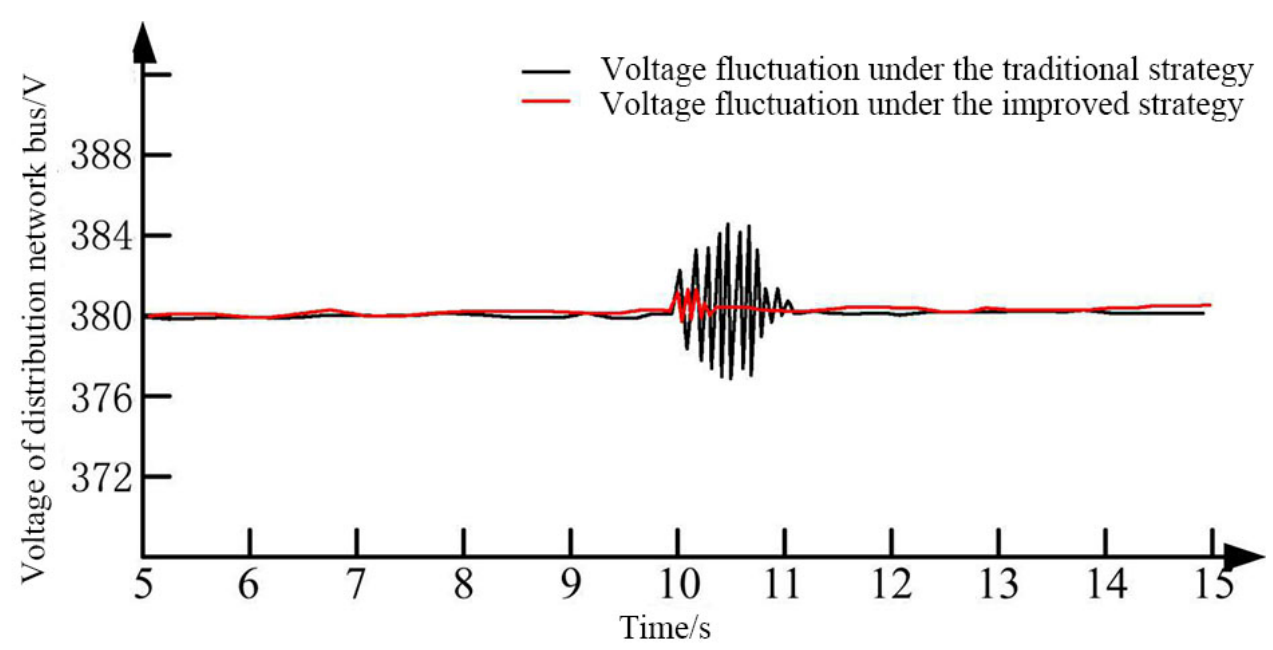

Figure 6 The bus voltage variation of the hybrid microgrid under two coordinated control strategies

As shown in Figure 6, before the change of light intensity, the photovoltaic output and gas turbine output was stable due to the stable light intensity, so the voltage of the distribution network bus in the microgrid was stable at $380 \mathrm{~V}$; at $10 \mathrm{~s}$, the light intensity dropped suddenly, the photovoltaic output also dropped sharply, and the gas turbine also provided output to guarantee the power needed by the load, during which the voltage of distribution network bus inevitably fluctuated; with the stability of light intensity, the photovoltaic output and gas turbine output became stable, and the bus voltage returned to the stability state again. It was seen from Figure 6 that the voltage fluctuation under the improved coordinated control strategy was smaller, and the duration was shorter. The reason was that the improved coordinated control strategy predicted the gas turbine and started the output ahead of time, which made the power switching cooperation of the two power generation systems smoother, more stable, and faster.

When the light intensity changed suddenly, the output power of the photovoltaic array also changed suddenly. To ensure the power demand of the load in the microgrid, the gas turbine started to provide output. However, due to the dynamic response delay, the output power of the gas turbine was unable to meet the requirements instantly. In this period, the power provided by the two power sources did not match the power required by the load; thus, the voltage of the distribution bus of the power grid lost stability, producing harmonics. The existence of harmonics could affect not only the microgrid but also the distribution network connected with the microgrid. As shown in Figure 7, the 5th and 7th harmonic content was $3.35 \%$ and $2.11 \%$ respectively under the traditional coordinated control strategy and $0.98 \%$ and $0.65 \%$, respectively, under the improved coordinated control strategy. It was seen that the harmonic content of the microgrid was lower under the improved coordinated control strategy. 


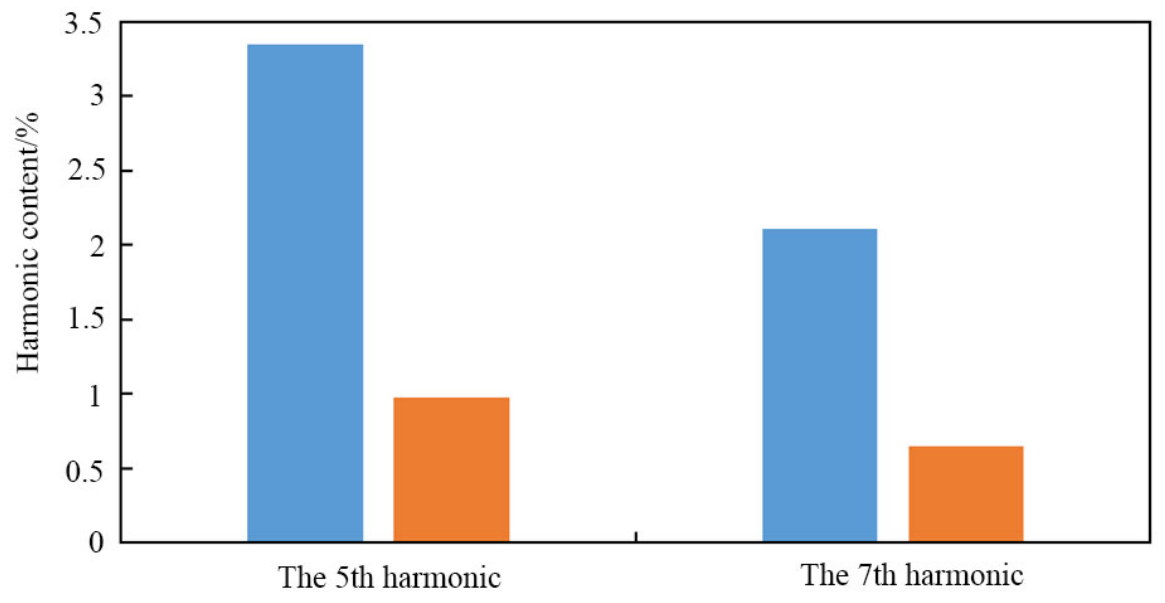

- The traditional coordinated control strategy n The improved coordinated control strategy

Figure 7 The 5th and 7th harmonic content in the microgrid under two coordinated control strategies

\section{CONCLUSION}

In this study, the power control framework of the photovoltaic power generation system and micro gas turbine power generation system in the hybrid microgrid was briefly introduced, the reference power of the micro gas turbine was predicted by using the prediction algorithm, the traditional coordinated control strategy of the microgrid was improved, and the simulation experiment was carried out on the two control strategies. The results are as follows: (1) when the light intensity dropped abruptly, the photovoltaic output power under two control strategies decreased sharply and coincided; the output power of the gas turbine gradually increased, and the increase was faster under the improved control strategy; (2) when the light intensity dropped suddenly, the distribution network voltage under the two control strategies fluctuated; the fluctuation under the improved control strategy was smaller and lasted for a shorter time; (3) when the sudden change of light intensity led to harmonics in the microgrid, the 5th and 7th harmonic content of the microgrid was smaller under the improved control strategy.

\section{REFERENCES}

[1] Xia, Y., W. Wei, Y. Peng, P. Yang, and M. Yu, Decentralized Coordination Control for Parallel Bidirectional Power Converters in a Grid-Connected DC Microgrid. IEEE Transactions on Smart Grid, 2018. 9(6): p. 6850-6861.

[2] Agrawal, A. and R. Gupta, Distributed coordination control of hybrid energy resources for power sharing in coupled hybrid DC/AC microgrid using paralleled IFCs/ILCs. IET Smart Grid, 2019. 2(1): p. 89-105. 
[3] Mohamed, E.A. and Y. Mitani, Enhancement the Dynamic Performance of Islanded Microgrid Using a Coordination of Frequency Control and Digital Protection. International Journal of Emerging Electric Power Systems, 2019. 20.

[4] Xia, Y., W. Wei, Y. Peng, P. Yang, and M. Yu, Decentralized Coordination Control for Parallel Bidirectional Power Converters in a Grid-Connected DC Microgrid. IEEE Transactions on Smart Grid, 2017: p. 1-1.

[5] Zhou, X., L. Zhou, Y. Chen, J.M. Guerrero, A. Luo, W. Wu, and L. Yang, A Microgrid Cluster Structure and Its Autonomous Coordination Control Strategy. IECON 2017 - 43rd Annual Conference of the IEEE Industrial Electronics Society. IEEE, 2018: p. 69-80.

[6] Lv, Z., Y. Xia, J. Chai, and M. Yu, Distributed Coordination Control Based on State-ofCharge for Bidirectional Power Converters in a Hybrid AC/DC Microgrid. Energies, 2018. 11(4): p. 1011.

[7] Shi, J., W. Huang, N. Tai, Q. Zhu, and D. Liu, Strategy to smooth tie-line power of microgrid by considering group control of heat pumps. Journal of Engineering, 2017. 2017(13): p. 2417-2422.

[8] Yang, P., Y. Xia, M. Yu, W. Wei, and Y. Peng, A Decentralized Coordination Control Method for Parallel Bidirectional Power Converters in a Hybrid AC-DC Microgrid. IEEE Transactions on Industrial Electronics, 2018. 65(8): p. 6217-6228.

[9] Xia, Y., Y. Peng, P. Yang, M. Yu, and W. Wei, Distributed Coordination Control for Multiple Bidirectional Power Converters in a Hybrid AC/DC Microgrid. IEEE Transactions on Power Electronics, 2016: p. 1-1.

[10] Tang, S., Z. Zeng, C. Cheng, H. Yang, R. Zhao, and Z. Lyu, Wireless coordination control of multi-functional grid-tied inverters in microgrid. Automation of Electric Power Systems, 2015. 39(9): p. 200-207.

[11] Lee, S.J., J.Y. Choi, H.J. Lee, and D.J. Won, Distributed Coordination Control Strategy for a Multi-Microgrid Based on a Consensus Algorithm. Energies, 2017. 10(7): p. 1017.

[12]Liang, B., L. Kang, J. He, F. Zheng, Y. Xia, Z. Zhang, Z. Zhang, G. Liu, and Y. Zhao, Coordination control of hybrid AC/DC microgrid. Journal of Engineering, 2019. 2019(16): p. 3264-3269.

[13]Zhang, J., Coordination control of multiple micro sources in islanded microgrid based on differential games theory. International Journal of Electrical Power \& Energy Systems, 2018. 97(APR.): p. 11-16.

[14]Cai, C., H. Liu, H. Zheng, F. Chen, L. Deng, and Q. Xu, Microgrid multi-source coordination optimal control based on multi-scenarios analysis. Journal of Engineering, 2017. 2017(13): p. 1457-1461.

[15]Zhang, J., Coordination Control of Multiple Micro Sources in Islanded Microgrid Based on Differential Games Theory. International Journal of Power and Energy Systems, 2017. 97.

[16] Chen, A., Coordination control and mode switching strategy for hybrid ac/dc microgrid with multi-bus structure. Automation of Electric Power Systems, 2018. 42(17): p. 175183.

[17] Sun, L., S. Han, W. Wu, and X. Guo, Stability coordination control of multi-resource integrated microgrid considering demand response. IOP Conference Series: Earth and Environmental Science, 2020. 446(4): p. 1-14. 
[18] Yoo, H.J., T.T. Nguyen, and H.M. Kim, Consensus-Based Distributed Coordination Control of Hybrid AC/DC Microgrids. IEEE Transactions on Sustainable Energy, 2019. 11(2): p. 629-639.

[19] Aryani, D.R. and H. Song, Voltage regulation in a stand-alone DC microgrid. IFACPapersOnLine, 2019. 52(4): p. 36-39.

[20] Karimi, H., G. Shahgholian, B. Fani, I. Sadeghkhani, and M. Moazzami, A protection strategy for inverter-interfaced islanded microgrids with looped configuration. Electrical Engineering, 2019. 101(3): p. 1059-1073. 
